Isabela Fernandes

Coelho Cunha ${ }^{1}$

Graduanda em Medicina -

Universidade Federal de Juiz de

Fora (UFJF), Membro do Grupo

de Pesquisa Ciência, Saúde e

Sociedade. E-mail:

Isabelafernandes2@homail.com

\section{Gabriela Sousa}

Leandro ${ }^{2}$

Graduanda em Medicina -

Universidade Federal de Juiz de

Fora (UFJF), Membro do Grupo

de Pesquisa Ciência, Saúde e

Sociedade. E-mail;

gabrielaleandro96@gmail.com

\section{Nicolas Emanuel \\ Oliveira Reis ${ }^{3}$}

Graduando em Medicina -

Universidade Federal de Juiz de

Fora (UFJF), Membro do Grupo

de Pesquisa Ciência, Saúde e

Sociedade. E-mail

nicolasemanuel@homail.com

\section{Larissa da Silva Torres}

França $^{4}$

Graduanda em Medicina -

Universidade Federal de Juiz de

Fora (UFJF), Membro do Grupo

de Pesquisa Ciência, Saúde e

Sociedade. E-mail E-mail:

larissasilvatf@gmail.com

\section{Waneska Alexandra} Alves $^{5}$

Doutora em Saúde Pública (ENSP/Fiocruz) E-mail:

waneska.alves@ufjf.edu.br

\section{MORTALIDADE POR ZOONOSES DE ACOMETIMENTO RESPIRATÓRIO EM MINAS GERAIS, BRASIL, 2010 A \\ 2019}

\author{
MORTALITY FOR RESPIRATORY ACCOMMODATION \\ ZOONOSES IN MINAS GERAIS, BRAZIL, 2010-2019
}

\section{RESUMO}

Introdução: As zoonoses representam um importante problema de saúde pública mundial. Conhecer a morbimortalidade em seres humanos causados por esses microrganismos é fundamental ao enfrentamento de emergências epidemiológicas. Objetivo: Estudar os registos de óbitos de em Minas Gerais, Brasil, por doenças respiratórias infecciosas cuja causa básica de morte tenha sido um microrganismo zoonótico. Metodologia: Conduziu-se estudo descritivo com dados secundários, não nominais e de domínio público disponibilizados pelo Ministério da Saúde. A fonte de dados foi o Sistema de Informação sobre Mortalidade. Utilizaram-se, para a análise dos dados, métodos de estatística e epidemiologia descritiva. Resultados e discussão: Foram identificados 243 óbitos $(0,3 \%$ do total) por doenças zoonóticas de acometimento respiratório. A taxa de mortalidade específica (por um milhão de habitantes) variou de 0,4 (2011) a 3,8 óbitos (2016). Os vírus representaram os agentes mais expressivos (80,2\%). Do total de óbitos, 56,0\% foram do sexo masculino, 39,1\% com idade entre 45-59 anos e $52,7 \%$ foram pessoas brancas. Apenas $50,2 \%$ foram investigados. Considerações finais: O delineamento descritivo, o uso de dados secundários e a escassez de estudos científicos sobre epidemiologia de zoonoses representaram as principais limitações desse estudo. Estudos ecoepidemiológicos sobre agentes zoonóticos são cruciais para o desenvolvimento de políticas públicas e o enfrentamento de endemias e epidemias.

PALAVRAS-CHAVE: Doenças respiratórias infecciosas. Zoonoses. Mortalidade.

\section{ABSTRACT}

Introduction: Zoonotic diseases represent a major problem for public health worldwide. Knowing about the morbidity and mortality in human beings caused by these microorganisms is essential when facing epidemiological emergencies. Aim: To study the death records in Minas Gerais, Brazil, due to infectious respiratory diseases whose the basic cause of death was a zoonotic microorganism. Methodology: A descriptive study was conducted with secondary, non-nominal and public domain data provided by the Ministry of Health. The source of data was the Mortality Information System. Descriptive epidemiology and statistics methods were used for data analysis. Results and 
discussions: 243 deaths $(0.3 \%$ of the total) due to zoonotic diseases of respiratory involvement were identified. The specific mortality rate (per one million inhabitants) ranged from 0.4 (2011) to 3.8 deaths (2016). Virus represented the most expressive agents $(80.2 \%)$. Of the total deaths, $56.0 \%$ were male, $39.1 \%$ were aged between $45-59$ years, and $52.7 \%$ had white skin color. Only $50.2 \%$ were investigated. Final considerations: The descriptive design, the use of secondary data and the scarcity of scientific studies on the epidemiology of zoonoses represented the main limitations of this study. Ecoepidemiological studies on zoonotic agents are crucial for the development of public policies to deal with endemic and epidemic diseases.

KEYWORDS: Infectious respiratory diseases, Zoonoses. Mortality.

\section{INTRODUÇÃO}

As doenças zoonóticas, também conhecidas como zoonoses, são enfermidades disseminadas dos animais para os seres humanos, a partir de diferentes agentes etiológicos, como vírus, bactérias, fungos e parasitas. Elas possuem elevada incidência mundial, visto que cerca de $3 / 4$ das doenças humanas emergentes e reemergentes possuem gênese zoonótica. Além disso, ocorrem, sobretudo, em nações em desenvolvimento. Essa conjuntura pode se relacionar às alterações comportamentais do homem durante os séculos, por exemplo, à domesticação e o convívio com animais silvestres, ao consumo de comidas exóticas, à globalização e à introdução de vetores em espaços urbanos devido ao desmatamento. Todas essas mudanças corroboram a modificação dos processos de transmissão de doenças (WALDMAN et al., 1996; KIMURA, 2002; ZANELLA, 2016).

Nesse sentido, observa-se a necessidade de ampliar e aprofundar os conhecimentos acerca da repercussão dos agentes zoonóticos no contexto da saúde animal e dos seres humanos, já que as zoonoses constituem grave problema de saúde pública. Essa urgência é comprovada pela conjuntura pandêmica da COVID-19, doença de acometimento respiratório relacionada ao vírus SARS-CoV-2 e que possui significativa morbimortalidade. Atualmente, são discutidas diferentes hipóteses sobre a sua origem, sendo uma delas associada à transmissão zoonótica direta de mamíferos para humanos, como os morcegos, que são apontados como reservatórios naturais do vírus, e os pangolins malaios (Manis javanica), uma vez que foi constatada a existência de cepas de SARSCoV-2 em populações dessa espécie, a qual é constantemente utilizada na gastronomia e na medicina tradicional chinesa. É nesse contexto que a prática da Saúde Única (One Health) se mostra imprescindível, buscando integrar homem, animal e meio ambiente como forma de compreender o surgimento de emergências em saúde, por exemplo, doenças emergentes e reemergentes, tais como as zoonoses, e as formas de controle e vigilância mais eficazes (ACOSTA et al., 2020; MACHALABA et al., 2021; WHO, 2021).

Somado a isso, na conjuntura das doenças infecciosas, em que mais da metade dessas morbidades são constituídas por zoonoses e das quais uma parcela importante acarreta sinais e sintomas respiratórios, é essencial o desenvolvimento de estudos clínicos, laboratoriais e epidemiológicos acerca dos agentes etiológicos que causam doenças e óbitos na população humana. Tal necessidade também é corroborada pela escassez de publicações científicas sobre zoonoses de acometimento respiratório, o que interfere de maneira importante nas ações públicas e privadas de vigilância, enfrentamento e controle 
de doenças emergentes e reemergentes que possuem potencial epidêmico (ACOSTA et al., 2020; RABBA et al., 2015).

Nesse cenário, vale ressaltar a importância dos sistemas de informação nas políticas nacionais de prevenção e promoção à saúde, como o Sistema de Informação sobre Mortalidade (SIM) do Ministério da Saúde (MS), o qual é apontado como padrão-ouro para pesquisas relacionadas à mortalidade e à letalidade de doenças e agravos. Por meio dos dados encontrados no SIM, são produzidas estatísticas e análises, como também indicadores epidemiológicos, os quais são fundamentais para o norteamento das ações promovidas pelos gestores do Sistema Único de Saúde (SUS). Entretanto, para que esses dados sejam produzidos e, posteriormente, utilizados de modo efetivo, é necessário que as Declarações de Óbito (DO), instrumento de coleta de dados do SIM, tenham seus campos preenchidos corretamente, como causas básicas e associadas de óbito. Essas causas, por sua vez, são codificadas e padronizadas segundo a 10 ${ }^{\underline{a}}$ edição da Classificação Internacional de Doenças (CID-10), criada pela Organização Mundial da Saúde (OMS), com o propósito de acompanhar os níveis de incidência e prevalência das enfermidades em diferentes escalas, desde a mundial até a municipal, para, a partir disso, comparar, formular e executar ações em saúde (FUNASA, 2001; MORAIS, COSTA, 2017).

O objetivo deste estudo foi analisar o perfil sociodemográfico e as causas básicas de morte dos indivíduos que evoluíram para o óbito em Minas Gerais, Brasil, entre 2010 a 2019, por doenças respiratórias infecciosas devido a microrganismos zoonóticos.

\section{MATERIAL E METODOS}

\section{Tipo de Estudo}

Foi conduzido delineamento metodológico descritivo com abordagem quantitativa e transversal. Utilizaram-se dados secundários e não nominais provenientes do Sistema de Informação sobre Mortalidade (SIM), disponibilizados pela Secretaria Estadual de Saúde de Minas Gerais (SES-MG), em domínio público. Os dados foram extraídos em 30 de março de 2021 e o período de estudo compreendeu óbitos ocorridos no interstício entre 01 de janeiro de 2010 a 31 de dezembro de 2019.

\section{Variáveis de Estudo}

Para alcançar os objetivos do estudo, foram analisadas variáveis sociodemográficas e clínicas como: ano e mês do óbito, sexo, idade (faixa etária), raça/cor, macrorregião de saúde de residência do óbito, causa básica de óbito (conforme CID-10 de agente infeccioso zoonótico) e investigação do óbito. Foram incluídos no estudo todos os registros de óbitos cujo CID-10 estava relacionado a doenças zoonóticas de acometimento pulmonar como causa básica de morte de pessoas residentes em municípios do estado de Minas Gerais, sendo eles: 1)Vírus: B33.4 (síndrome cardiopulmonar por Hantavírus), J09 (influenza/gripe devido a vírus identificado da gripe aviária), J12.0 (pneumonia viral devido a Adenovírus), J12.1 (pneumonia devido a Vírus Respiratório Sincicial) e J12.2 (pneumonia devido a Parainfluenza); 2) Bactérias: A42.0 (actinomicose pulmonar); 3) Fungos e Parasitas: B46.0 (mucormicose pulmonar), B58.3 (toxoplasmose pulmonar), B38.2 (coccidioidomicose pulmonar 
não especificada), B39.0 (histoplasmose pulmonar aguda por Histoplasma capsulatum), B39.1 (histoplasmose pulmonar crônica por Histoplasma capsulatum), B39.2 (histoplasmose pulmonar não especificada por Histoplasma capsulatum), B40.0 (blastomicose pulmonar aguda), B40.1 (blastomicose pulmonar crônica), B40.2 (blastomicose pulmonar não especificada) e B45.0 (criptococose pulmonar).

Foram excluídos registros de pessoas que residiam em municípios de outra unidade federada ou cuja causa básica de morte não estivesse claramente relacionada a agente infeccioso zoonótico. Também não foram analisados CIDs de doenças zoonóticas que não possuíam registros no SIM no local e período estudados.

Cabe ressaltar que a tuberculose causada pelo Mycobacterium bovis pode ser considerada como uma importante zoonose de acometimento pulmonar, porém os códigos do CID-10 para tuberculose no SIM não especificam o agente etiológico. Assim, para evitar superestimativas, os códigos A15 e A16 (tuberculoses causadas por outros agentes etiológicos ou inespecíficas, respectivamente) foram excluídos do estudo.

\section{Análise de Dados}

Para a análise dos dados utilizaram-se técnicas de estatística descritiva como a análises de medidas de tendência central, frequência (n) e proporção (\%). Foram também calculadas taxas de mortalidade específica ( $\mathrm{n}$ ำ de óbitos por causa específica dividido pela população residente estimada e exposta ao risco de morrer, multiplicado por um milhão). Os dados populacionais foram obtidos nas estimativas do Instituto Brasileiro de Geografia e Estatística (IBGE), disponibilizados no portal do Tabnet da SES-MG. Utilizaram-se os softwares Microsoft Excel® e Epi InfoTM 7.2 (Centro de Controle de Doenças Norte Americano).

\section{ASPECTOS ÉTICOS DA PESQUISA}

O estudo dispensou apreciação pelo Comitê de Ética em Pesquisa institucional por utilizar dados secundários, não nominais e disponíveis em domínio público na internet (http://vigilancia.saude.mg.gov.br/), porém as resoluções № 466/12 e 510/16 do Conselho Nacional de Saúde (CONEP/MS) foram rigorosamente respeitadas.

\section{RESULTADOS}

As zoonoses podem comprometer vários sítios sistêmicos, sendo o aparelho respiratório um deles, e, em alguns casos, podem ter agentes etiológicos potenciais causadores de endemias ou epidemias, a exemplo da COVID-19, tornando-se problemas importantes de saúde pública mundial (WHO, 2020). Estima-se que as zoonoses sejam responsáveis por 2,5 bilhões de casos de doenças humanas e por mais de 2,7 milhões de mortes humanas por ano, no mundo (JUNIOR, 2018). Para o presente estudo, foi realizada uma vasta investigação da literatura científica nacional e internacional, o que permitiu constatar uma escassez de estudos, tanto descritivos quanto analíticos, envolvendo a mortalidade por zoonoses.

Dos 103 códigos apresentados no banco de dados do SIM como causa básica de morte por doenças infecciosas respiratórias, apenas 15 (14,6\%) estavam 
associados a agentes infecciosos zoonóticos, cujo principal foco de infecção incluía o aparelho respiratório humano. Dos 85.108 óbitos por doenças infecciosas respiratórias, registrados de 2010 a 2019, 243 (0,3\%) foram mortes por doenças zoonóticas de acometimento no sistema respiratório. Vale destacar que, segundo relatório do Global Burden of Disease (2015), as infecções do trato respiratório inferior são responsáveis por uma taxa de mortalidade padronizada por idade de 41,6 óbitos/100 mil habitantes.

A taxa de mortalidade específica (por um milhão de habitantes) variou ao longo da série histórica conforme gráfico 1. A amplitude da taxa foi de 0,4 (2011) a 3,8 (2016) óbitos. Dentre os grupos de agentes etiológicos, os vírus representaram os agentes mais expressivos, sendo responsáveis por 80,2\% (195) dos óbitos, com destaque para o vírus Influenza ( $81,5 \%$ dos casos referentes ao grupo). Isso se deve, provavelmente, à vigilância de doenças respiratórias centrada na Influenza, realizada no Brasil pelo Ministério da Saúde e pelas secretarias estaduais de saúde. A literatura corrobora a importância da influenza no cenário de morbimortalidade mundial, uma vez que, em 2015, a infecção por influenza fomentou um total estimado de 83,1 mil óbitos (GBD, 2015). Desde 1996, foram relatados mais de 14 subtipos de influenza aviária, sendo os mais virulentos: H1N1, H5N1 e H7N9. Em 2016, a influenza por vírus H5N1 afetou 16 países, causando 846 casos e 449 mortes (LI; CAO, 2016). Ademais, um estudo realizado na China com 143 pacientes confirmados com H7N9 apresentou letalidade expressiva de 37,1\% (53 óbitos) (YANG et al., 2019). Diversos estudos reforçam o potencial patogênico dos vírus emergentes.

Gráfico 1. Série histórica dos registros de óbitos e taxas de mortalidade especifica por agente zoonótico, 2010 a 2019. Minas Gerais, Brasil.

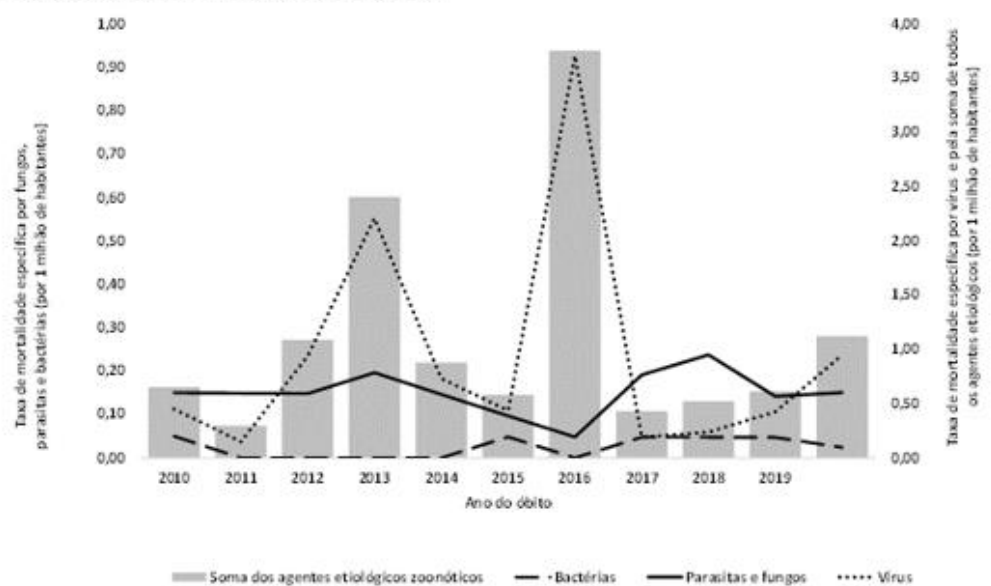

Fonte: Sistema de Informação de Mortalidade. Ministério da Saúde do Brasil.

O grupo dos fungos e parasitas foi responsável por 17,7\% (43) dos óbitos. Dentre as infecções fúngicas zoonóticas, a criptococose pulmonar é a mais frequentemente relatada, sendo uma infecção oportunista de alta letalidade em indivíduos imunocomprometidos e cuja forma pulmonar foi encontrada como causa básica de morte mais recorrente no grupo dos fungos e parasitas, representando $32,6 \%$ das mortes. Estudos apontam para incidência de 0,9 casos/100 mil habitantes, sendo a prevalência da doença mais bem conhecida nos soropositivos para HIV/Aids (CHANG; SORRELL; CHEN, 2015; FANG; FA; 
LIAO, 2015). Nesse âmbito, o pequeno número de óbitos por criptococose pulmonar registrados no SIM, para Minas Gerais, pode ser justificado pelo fato de a doença se comportar de forma pouco expressiva na população imunocompetente em geral, além de não estar incluída, até o ano de 2020 (BRASIL, 2020), na Lista Nacional de Notificação Compulsória de doenças, agravos e eventos de saúde pública.

As bactérias, por sua vez, representaram apenas 2,1\% (5) dos óbitos, com a mortalidade sendo $100,0 \%$ decorrente da actinomicose, uma doença crônica rara causada por um grupo de bactérias anaeróbicas (Actinomyces spp.) grampositivas que normalmente colonizam a boca, o cólon e o trato urogenital (VALOUR et al., 2014). A identificação de poucos registros, referentes à forma pulmonar dessa doença, está em concordância com o caráter raro da enfermidade, bem como com o fato dela não ser de notificação compulsória.

Em relação à distribuição das taxas de mortalidade específicas por zoonoses segundo macrorregião de saúde (Macro), a maior taxa de mortalidade por bactérias se deu na Macro-Noroeste ( 0,2 óbitos) e por vírus ocorreu na MacroOeste (2,7 óbitos). A taxa de mortalidade por parasitas e fungos mostrou-se mais expressiva nas Macros-Triângulo do Sul e Vale do Aço, sendo a última a única macrorregião em que, proporcionalmente, a taxa de mortalidade por parasitas e fungos foi predominante (66,7\%) (Gráfico 2). Dada as lacunas científicas referentes à epidemiologia das zoonoses em Minas Gerais, os dados do gráfico 2 precisam ser melhor analisados à luz dos estudos e inquéritos ecológicos que considerem contextos ambientais, humanos e animais na manifestação da morbimortalidade no estado. Assim, é necessário que as instituições governamentais e de pesquisa promovam tais estudos, para que evidências científicas possam ser produzidas.

Gráfico 2. Taxas de mortalidade especifica por agente zoonótico, 2010 a 2019. Minas Gerais, Brasil.

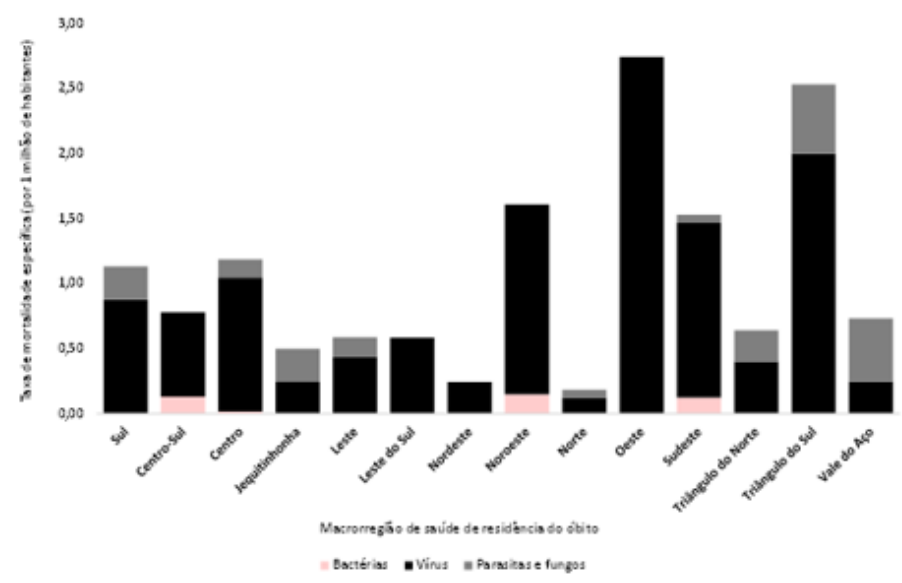

Fonte: Sistema de Informação de Mortalidade. Ministério da Saúde do Brasil.

Quanto às características sociodemográficas dos óbitos, 56,0\% foram do sexo masculino, 39,1\% ocorreram na faixa etária de 45 a 59 anos, com média de 52,2 anos para pessoas do sexo masculino (desvio padrão $=19,9$ ) e 51,4 anos para sexo feminino (desvio padrão = 21,2). Para distribuição dos óbitos por raça/cor, $52,7 \%$ foram pessoas brancas (Tabela 1 ). As faixas etárias mais evidentes indicam que a população economicamente ativa é a mais afetada pelas doenças 
zoonóticas analisadas, o que pode estar relacionado às condições de trabalho e de vida. Em relação ao sexo, a literatura cientifica aponta para maior morbimortalidade de doenças respiratórias no sexo masculino (DIAS et al., 2020; LAURENTI; JORGE; GOTLIEB, 2005). Quanto à maior frequência da raça/cor branca, é importante destacar que, entretanto, pessoas pretas e pardas são maioria no estado de Minas Gerais, segundo o Instituto Brasileiro de Geografia e Estatística (2011), o que reflete a importância de estudos epidemiológicos envolvendo essa variável. Junto a isso, a ausência de registros com raça/cor indígena pode estar relacionada à subnotificação e/ou ao registro de má qualidade, dado o significativo número de casos sem informação para raça/cor $(11,5 \%)$ (Tabela 1).

Para o grupo de agentes etiológicos referentes aos vírus, com grande destaque para o vírus influenza, foi encontrada predominância do sexo masculino e da faixa etária de 45 a 59 anos (Tabela 1). Um estudo que analisou o caráter global de mortalidade por influenza apresentou discordância quanto à faixa etária, indicando que, do total de mortes respiratórias anuais associadas à influenza, $67 \%$ estavam entre pessoas com 65 anos ou mais (PAGET et al., 2019). Além disso, Yang et al. (2019) mostraram que, em pacientes hospitalizados com o vírus H7N9 (influenza identificado da gripe aviária), a maior taxa de letalidade ocorreu no grupo com mais de 63,5 anos, indicando, ainda, maioria das mortes para o sexo feminino, o que, também, opõe-se aos achados deste estudo. Freitas, Francisco e Donalisio (2013) mostraram que, para o estado de São Paulo, a maior subnotificação de diagnóstico de influenza ocorreu em maiores de 60 anos. Nesse âmbito, o resultado deste estudo acerca das idades pode estar relacionado à subnotificação, além da possível influência das campanhas anuais de vacinação contra a gripe que contemplam a população idosa, iniciadas em 1999 (BRASIL, 2013).

Mortalidade por fungos com predominância para o sexo masculino e faixas etárias adultas foi descrita por Soares et al. (2019). Dentre o grupo das bactérias, representado pela actinomicose, também, há maior acometimento do sexo masculino, estando em concordância com dados da literatura, os quais descrevem infecção três vezes maior nesse grupo em relação ao sexo feminino (VALOUR et al., 2014). Isso pode decorrer, parcialmente, da higiene bucal precária e/ou da maior incidência de trauma facial em homens, resultando em doenças dentárias e faciais, o que facilita a infecção do patógeno causador, tendo em vista que a boca é um dos sítios de colonização da Actinomyces spp. (VALOUR et al., 2014; MABEZA; MACFARLANE, 2003; LAURENTI; JORGE; GOTLIEB, 2005).

Quanto à investigação dos óbitos, em apenas 50,2\% houve procedimentos investigativos para definir a causa básica da morte (Tabela 1). O pequeno percentual que passa por processo investigativo compromete fortemente a identificação da etiologia das doenças e das mortes, já que o percentual de códigos garbage (códigos lixos) é elevado no SIM. 
Tabela 1. Perfil sociodemográfico dos óbitos por zoonoses de acometimento respiratório, Minas Gerais, Brasil, 2010 a 2019.

\begin{tabular}{|c|c|c|c|c|}
\hline \multirow{3}{*}{ Variáveis } & \multicolumn{3}{|c|}{ Grupos de agentes etiológicos } & \multirow{3}{*}{$\begin{array}{c}\text { Total } \\
\mathrm{N}^{\circ} \text { óbitos (\%) }\end{array}$} \\
\hline & Bactéria & Vírus & $\begin{array}{l}\text { Fungos e } \\
\text { Parasitas }\end{array}$ & \\
\hline & $\begin{array}{c}\mathbf{N}^{0} \text { óbitos } \\
(\%)\end{array}$ & $\begin{array}{c}\mathbf{N}^{0} \text { óbitos } \\
(\%)\end{array}$ & $\mathrm{N}^{0}$ óbitos & \\
\hline \multicolumn{5}{|l|}{ Sexo } \\
\hline Masculino & $3(2,2)$ & $102(75,0)$ & $31(22,8)$ & $136(56,0)$ \\
\hline Feminino & $2(1,9)$ & $93(86,9)$ & $12(11,2)$ & $107(44,0)$ \\
\hline \multicolumn{5}{|l|}{ Faixa etária } \\
\hline $0-17$ anos & $0(0,0)$ & $15(100,0)$ & $0(0,0)$ & $15(6,2)$ \\
\hline 18-21 anos & $0(0,0)$ & $2(100,0)$ & $0(0,0)$ & $2(0,8)$ \\
\hline $21-45$ anos & $1(1,9)$ & $44(83,0)$ & $8(15,1)$ & $53(21,8)$ \\
\hline $45-59$ anos & $1(1,1)$ & $79(83,1)$ & $15(15,8)$ & $95(39,1)$ \\
\hline $60-74$ anos & $2(4,6)$ & $32(72,7)$ & $10(22,7)$ & $44(18,1)$ \\
\hline$>74$ anos & $1(2,9)$ & $23(67,6)$ & $10(29,4)$ & $34(14,0)$ \\
\hline \multicolumn{5}{|l|}{ Raça/Cor } \\
\hline Branca & $2(1,6)$ & $103(80,4)$ & $23(18,0)$ & $128(52,7)$ \\
\hline Preta & $1(5,3)$ & $15(78,9)$ & $3(15,8)$ & $19(7,8)$ \\
\hline Amarela & $0(0,0)$ & $2(100,0)$ & $0(0,0)$ & $2(0,8)$ \\
\hline Parda & $2(3,0)$ & $52(78,8)$ & $12(18,2)$ & $66(27,2)$ \\
\hline Indígena & $0(0,0)$ & $0(0,0)$ & $0(0,0)$ & $0(0,0)$ \\
\hline Sem informação & $0(0,0)$ & $23(82,1)$ & $5(17,9)$ & $28(11,5)$ \\
\hline \multicolumn{5}{|l|}{$\begin{array}{l}\text { Investigação do } \\
\text { óbito }\end{array}$} \\
\hline Sim & $1(0,8)$ & $116(95,1)$ & $5(4,1)$ & $122(50,2)$ \\
\hline Não & $0(0,0)$ & $29(59,2)$ & $20(40,8)$ & $49(20,2)$ \\
\hline Sem informação & $4(5,6)$ & $50(69,4)$ & $18(25,0)$ & $72(29,6)$ \\
\hline
\end{tabular}

Fonte: Sistema de Informação de Mortalidade. Ministério da Saúde do Brasil.

O presente estudo traz uma abordagem inovadora ao caracterizar o perfil sociodemográfico e a mortalidade por zoonoses que acometem o sistema respiratório, servindo de base para estudos complementares que possam oferecer evidências cientificas consistentes acerca da epidemiologia da morbimortalidade por zoonoses em Minas Gerais com foco na Saúde Única (One Health). Dentre as limitações deste estudo, destacam-se o uso de dados secundários e a escassez de estudos científicos disponibilizados na literatura mundial, acerca da epidemiologia das zoonoses de acometimento respiratório. Vale destacar que o sistema de informação utilizado nesta pesquisa está consolidado como padrão-ouro para estudos sobre mortalidade no Brasil, ainda que a qualidade no preenchimento da Declaração de Óbito possa ser uma fragilidade.

\section{CONSIDERAÇÕES FINAIS}

A mortalidade por zoonoses de acometimento respiratório, em Minas Gerais, entre 2010 e 2019, tem como perfil o óbito predominantemente por agentes virais, acometendo, sobretudo, pessoas do sexo masculino, adultas e da raça/cor branca, residentes na macrorregião Oeste do estado. As lacunas científicas fortalecem a recomendação para o aprofundamento científico referente ao tema deste artigo, com a realização de mais estudos clínicos e ecoepidemiológicos. Assim, as zoonoses representam uma ameaça não apenas à saúde dos animais e dos seres humanos, mas também à segurança sanitária mundial. Nessa perspectiva, o conceito de Saúde Única (One Health) possibilita 
uma abordagem colaborativa e multisetorial à acepção de saúde ao estabelecer que a saúde das pessoas está intimamente relacionada à saúde dos animais e ao ambiente compartilhado. Objetiva-se, atualmente, alcançar a saúde ideal a todos esses entes, por meio de ações multiprofissionais na busca pelo cuidado dos seres humanos, dos animais e do meio ambiente, minimizando a incidência e a mortalidade por zoonoses no mundo. Os estudos ecoepidemiológicos sobre agentes zoonóticos são cruciais para o desenvolvimento de diretrizes e de políticas públicas efetivas para o enfrentamento das endemias atuais, bem como das epidemias e pandemias futuras.

\section{REFERÊNCIAS}

ACOSTA, Andre Luis et al. Interfaces à transmissão e spillover do coronavírus entre florestas e cidades. Estudos Avançados, v. 34, n. 99, p. 191-208, 2020. Disponível em: <https://www.scielo.br/j/ea/a/GNgmbbDG5t6rtLwxkvbNq4k/>. Acesso em: 06 abr. 2021.

WALDMAN, Eliseu Alves et al. Gastroenterites e infecções respiratórias agudas em crianças menores de 5 anos em área da região Sudeste do Brasil, 19861987: I-Infecções respiratórias agudas. Revista de Saúde Pública, v. 30, p. 553563, $1996 . \quad$ Disponível em: <https://www.scielo.br/j/rsp/a/Fh864wdF9K4ghYFsdfhWDxL/?lang=pt>. Acesso em: 09 abr. 2021.

BRASIL. Ministério da Saúde. Portaria № 264, de 17 de fevereiro de 2020. Dispõe sobre a inclusão da doença de Chagas crônica, da criptococose, da esporotricose humana e da paracoccidioidomicose na Lista Nacional de Notificação Compulsória de doenças, agravos e eventos de saúde pública. Brasília: Ministério da Saúde, 2020. Disponível em: <https://bvsms.saude.gov.br/bvs/saudelegis/gm/2020/prt0264_19_02_2020.ht $\mathrm{ml}$. Acesso em: 07 abr. 2021.

BRASIL. Ministério da Saúde. Secretaria de Vigilância em Saúde. Departamento de Vigilância Epidemiológica. Programa Nacional de Imunizações (PNI): 40 anos. Brasília: Ministério da Saúde, 2013. Disponível em: $<$ https://bvsms.saude.gov.br/bvs/publicacoes/programa_nacional_imunizacoes _pni40.pdf>. Acesso em 09 abr. 2021.

CHANG C. C.; SORRELL T. C.; CHEN S. C. Pulmonary Cryptococcosis. Semin Respir Crit Care Med., v. 36, p. 681-91, 2015. Disponível em: <https://pubmed.ncbi.nlm.nih.gov/26398535/>. Acesso em: 07 abr. 2021.

DIAS, Fellipe Leonardo Torres et al. Doenças respiratórias no Triângulo Mineiro: Análise epidemiológica e projetiva com a pandemia de COVID-19. J. Health Biol Sci., v. 8, n. 1., p. 1-6, 2020. Disponível em: $<$ https://periodicos.unichristus.edu.br/jhbs/article/view/3219>. Acesso em 08 abr. 2021.

FANG, Wei; FA, Zhenzong; LIAO, Wanqing. Epidemiology of Cryptococcus and cryptococcosis in China. Fungal Genet Biol., v. 78, p. 7-15, 2015. Disponível em: $<$ https://www.sciencedirect.com/science/article/pii/S1087184514002023?via\%3 Dihub>. Acesso em: 08 abr. 2021. 
FREITAS, André Ricardo Ribas; FRANCISCO, Priscila M. S. Bergamo; DONALISIO, Maria Rita. Mortality Associated with Influenza in Tropics, State of São Paulo, Brazil, from 2002 to 2011: The Pre-Pandemic, Pandemic, and PostPandemic Periods. Influenza Research and Treatment, v. 2013, p. 1-10, 2013. Disponível em: <https://www.hindawi.com/journals/irt/2013/696274/>. Acesso em 08 abr. 2021.

GBD 2015. Global, regional, and national life expectancy, all-cause mortality, and cause-specific mortality for 249 causes of death, 1980-2015: a systematic analysis for the Global Burden of Disease Study 2015. Lancet, v. 388, p. 14591544, $2016 . \quad$ Disponível em: <https://www.thelancet.com/action/showPdf?pii=S0140-6736\%2816\%29310121>. Acesso em: 07 abr. 2021.

INSTITUTO BRASILEIRO DE GEOGRAFIA E ESTATÍSTICA - IBGE. Censo Demográfico 2010: Características da população e dos domicílios, resultados do universo. Rio de Janeiro: IBGE, 2011. Disponível em: <https://biblioteca.ibge.gov.br/visualizacao/periodicos/93/cd_2010_caracteristic as_populacao_domicilios.pdf>. Acesso em 08 abr. 2021.

JUNIOR, Jorge Granja de Oliveira. Contribuição à vigilância da Influenza Equina no Pantanal Sul-Matogrossense. 2018. Tese (Doutorado em Ciência Animal) Universidade Federal de Mato Grosso do Sul, Campo Grande, 2018. Disponível em: <http://www.alice.cnptia.embrapa.br/alice/handle/doc/1106682>. Acesso em: 05 abr. 2021.

KIMURA, Leda Maria Silva. Principais zoonoses. Animais de laboratório: criação e experimentação. Rio de Janeiro: Fiocruz, 2002.

LAURENTI, Ruy.; JORGE, Maria Helena Prado de Mello; GOTLIEB, Sabina Léa Davidson. Perfil epidemiológico da morbimortalidade masculina. Ciênc. saúde coletiva, Rio de Janeiro, v. 10, n. 1, p. 35-46, mar. 2005. Disponível em: $<$ http://www.scielo.br/scielo.php?script=sci_arttext\&pid=S1413-

$81232005000100010 \&$ Ing=en\&nrm=iso >. Acesso em 08 abr. 2021.

LI Hui; CAO Bin. Pandemic and Avian Influenza A Viruses in Humans: Epidemiology, Virology, Clinical Characteristics, and Treatment Strategy. Clin Chest Med., v. 38, n.1, p. 59-70, 2017. Disponível em: <https://pubmed.ncbi.nlm.nih.gov/28159162/>. Acesso em: 08 abr. 2021. MABEZA, G. F., MACFARLANE, J. Pulmonary actinomycosis. Eur Respir J., v. 21, n. 3, p. 545-551, mar. 2003. Disponível em: <https://erj.ersjournals.com/content/21/3/545.long>. Acesso em 08 abr. 2021.

MACHALABA, Catherine Machalaba et al. Applying a One Health Approach in Global Health and Medicine: Enhancing Involvement of Medical Schools and Global Health Centers. Annals of global health, v. 87, n. 1, 2021. Disponível em: <https://pubmed.ncbi.nlm.nih.gov/33816135/>. Acesso em: 09 abr. 2021.

MORAIS, Rinaldo Macedo de; COSTA, André Lucirton. An Evaluation of the Brazilian Mortality Information System. Saúde em Debate, v. 41, p. 101-117, 2017. 
<https://www.scielo.br/j/sdeb/a/FJXQhtgNM3S5qvGHNfLMk3Q/?lang=en\&form at=pdf>. Acesso em: 09 abr. 2021.

PAGET, John et al. Global mortality associated with seasonal influenza epidemics: New burden estimates and predictors from the GLaMOR Project. Journal of global health, v. 9, n. 2, p. 1-12, dec. 2019. Disponível em: $<$ https://www.ncbi.nlm.nih.gov/pmc/articles/PMC6815659/>. Acesso em 08 abr. 2021.

RABAA, Maia A. et al. The Vietnam Initiative on Zoonotic Infections (VIZIONS): a strategic approach to studying emerging zoonotic infectious diseases. Ecohealth, v. 12, n. 4, p. 726-735, 2015. Disponível em: <https://pubmed.ncbi.nlm.nih.gov/26403795/>. Acesso em: 09 abr. 2021.

SOARES, Emmanuel Alves et al. Mortality by cryptococcosis in Brazil from 2000 to 2012: A descriptive epidemiological study. PLoS Negl Trop Dis, v. 13, n. 7, p. 1-17, jul. 2019. Disponível em: <https://www.arca.fiocruz.br/handle/icict/35495>. Acesso em: 09 abr. 2021

VALOUR, Florent Valour et al. Actinomycosis: etiology, clinical features, diagnosis, treatment, and management. Infection and Drug Resistance, v. 2014, n. 7 , p. 183-197, jul. 2014. Disponível em: $<$ https://www.dovepress.com/actinomycosis-etiology-clinical-featuresdiagnosis-treatment-and-manag-peer-reviewed-article-IDR>. Acesso em: 09 abr. 2021.

WORLD HEALTH ORGANIZATION - WHO. WHO-convened global study of origins of SARS-CoV-2: China Part. WHO, 2021. Disponível em: $<$ https://www.who.int/publications/i/item/who-convened-global-study-of-originsof-sars-cov-2-china-part>. Acesso em: 07 abr. 2021.

WORLD HEALTH ORGANIZATION - WHO. Zoonosis. 2020. Disponível em: $<$ https://www.who.int/news-room/fact-sheets/detail/zoonoses>. Acesso em: 08 abr. 2021.

YANG, Yang et al. Clinical indices and mortality of hospitalized avian influenza A (H7N9) patients in Guangdong, China. Chin Med J (Engl), v. 132, n. 3, p. 302310 , 2019. Disponível

em: <https://journals.Iww.com/cmj/Fulltext/2019/02050/Clinical_indices_and_mortali ty_of_hospitalized.7.aspx>. Acesso em: 07 abr. 2021.

ZANELLA, Janice Reis Ciacci. Zoonoses emergentes e reemergentes e sua importância para saúde e produção animal. Pesquisa agropecuária brasileira, v. 51, p. 510-519, 2016. Disponível em: <https://www.scielo.br/j/pab/a/LjPRt7VpRQdW3cWTY3KZ4Pj/?lang=pt>.

Acesso em: 07 abr. 2021. 